\title{
UNIFORM ABSOLUTE CONTINUITY IN SPACES OF SET FUNCTIONS
}

\author{
JAMES D. STEIN, JR.
}

ABSTRACT. Let $X$ be a regular topological space, $K$ a collection of bounded regular measures defined on the Borel sets of $X$. The following conditions are equivalent.

(1) Let $M(X)$ denote the Borel measures, $M(X)^{+}$the nonnegative members of $M(X)$. There is a $\lambda \in M(X)^{+}$such that $K$ is uniformly $\lambda$-continuous.

(2) If $\left\{U_{n} \mid n=1,2, \ldots\right\}$ is a di sjoint sequence of open set $s$, then $\lim _{n \rightarrow \infty} \mu\left(U_{n}\right)=0$ uniformly for $\mu \in K$.

(3) If $E$ is a Borel subset of $X$ and $\epsilon>0$, there is a compact set $F \subseteq$ $E$ such that $|\mu|(E \sim F)<\epsilon$ for $\mu \in K$.

(4) If $\left\{E_{n} \mid n=1,2, \ldots\right\}$ is a disjoint sequence of Borel sets, then $\lim _{n \rightarrow \infty} \mu\left(E_{n}\right)=0$ uniformly for $\mu \in K$.

Throughout this paper, $X$ will denote a regular topological space (one which satisfies the separation axioms $T_{1}$ and $T_{3}$ ). A bounded set function $\mu$ defined on the Borel subsets of $X$ is said to be regular if, for each Borel set $E \subseteq X$ and $\epsilon>0$ we can find a compact set $K \subseteq E$ such that $|\mu(E \sim K)|$ $<\epsilon$. If $K$ is a collection of set functions defined on the Borel sets and $\lambda$ is a nonnegative set function also defined on the Borel sets, we say that $K$ is uniformly absolutely continuous with respect to $\lambda$ if, for every $\epsilon>0$, there is a $\delta>0$ such that if $E$ is a Borel set with $\lambda(E)<\delta$, then $|\mu(E)|<\epsilon$ for each $\mu \in K$.

In [1], Grothendieck gives several conditions equivalent to uniform absolute continuity for sets of bounded regular measures defined on the Borel sets of a locally compact Hausdorff space. In this paper we shall extend Grothendieck's theorem to sets of bounded regular additive set functions defined on the Borel sets of a regular topological space.

If $\mu$ is a set function, $|\mu|$ will denote the total variation of $\mu$. We let $M(X)$ denote the Borel measures, and $M(X)^{+}$denote the nonnegative members of $M(X)$.

Lemma. (1) $|\mu| \in M(X)$.

Received by the editors December 5, 1972 and, in revised form, May 23, 1974. AMS (MOS) subject classifications (1970). Primary $28 \mathrm{~A} 10$. 
(2) For $\mu \in M(X)$ and $E$ a Borel subset of $X,|\mu|(E) \leq \pi \sup \{|\mu(F)| \mid$ $E \supset F, F$ Borel\}.

(3) For $\mu \in M(X)$ and $U$ an open subset of $X$, there is an open subset $V$ of $U$ such that $|\mu(V)| \geq 1 / 4|\mu|(U)$.

Proof. The proof of (1) is elementary, and (2) is a consequence of the fact that, given a finite set $\left\{\alpha_{1}, \ldots, \alpha_{n}\right\}$ of complex numbers, there is a subset $\alpha_{n}, \ldots, \alpha_{n_{p}}$ of them such that $\sum_{k=1}^{n}\left|\alpha_{k}\right| \leq \pi\left|\sum_{j=1}^{p} \alpha_{n_{j}}\right| \cdot$. (3) can easily be deduced from (2) and regularity. Q.E.D.

We now present the main result of this paper.

Theorem. Let $K \subset M(X)$. The following conditions are equivalent.

(a) There is a $\lambda \in M(X)^{+}$such that $K$ is uniformly $\lambda$-continuous.

(b) If $\left\{U_{n} \mid n=1,2, \ldots\right\}$ is a disjoint sequence of open subsets of $X$, then $\lim _{n \rightarrow \infty} \mu\left(U_{n}\right)=0$ uniformly for $\mu \in K$.

(c) If $E$ is a Borel subset of $X$ and $\epsilon>0$, there is a compact set $F \subseteq$ E such that $|\mu|(E \sim F)<\epsilon$ for all $\mu \in K$.

(d) If $\left\{E_{n} \mid n=1,2, \ldots\right\}$ is a disjoint sequence of Borel subsets of $X$, then $\lim _{n \rightarrow \infty} \mu\left(E_{n}\right)=0$ uniformly for $\mu \in K$.

Proof. Since $K$ satisfies one of the conditions (a), (b), (c), and (d) iff $\{\nu \in M(X)|\exists \mu \in K \ni 0 \leq \nu \leq| \mu \mid\}$ satisfies the same condition, we may assume that $K \subset M(X)^{+}$and that $\nu \in K$ whenever $\exists \mu \in K \ni 0 \leq \nu \leq \mu$.

(a) $\Rightarrow$ (b): This is clear, since $\lim _{n \rightarrow \infty} \lambda\left(U_{n}\right)=0$.

(b) $\Rightarrow$ (c): We first show that (b) implies $\left(c^{\prime}\right)$ : If $F$ is a compact subset of $X$ and if $\epsilon>0$, then there is an open set $V \supseteq F$ such that $\mu(V \sim F)<\epsilon$ for all $\mu \in K$.

Suppose (b) holds but ( $\left.c^{\prime}\right)$ does not. Let $\epsilon_{1}=0$ and $\epsilon_{n}=\epsilon$ if $n \geq 2$. We shall obtain a contradiction by constructing a sequence $\left\{\mu_{n} \mid n=1,2, \ldots\right\} \subseteq$ $K$ and a sequence $\left\{U_{n} \mid n=1,2, \ldots\right\}$ of open sets such that

(1) $\mu_{n}\left(U_{n}\right) \geq \epsilon_{n}$

(2) if $n \neq m, \bar{U}_{n} \cap \bar{U}_{m}=\varnothing$,

(3) $\bar{U}_{n} \subset X \sim F$.

Let $U_{1}=\varnothing$, and let $\mu_{1}$ be any member of $K$. Suppose $\mu_{1}, \ldots, \mu_{n}$ and $U_{1}$, $\ldots, U_{n}$ have been chosen in accordance with (1), (2), and (3). $X \sim \bigcup_{j=1}^{n} \bar{U}_{j}$ is an open set containing $F$, and so by the compactness of $F$ and the regularity of $X$ there is an open set $U_{n+1}$ such that $F \subset U_{n+1} \subset \bar{U}_{n+1} \subset X \sim$ $\bigcup_{j=1}^{n} \bar{U}_{j}$. By our assumption, there is a $\mu_{n+1} \in K$ such that $\mu_{n+1}\left(U_{n+1} \sim F\right)$ $\geq \epsilon=\epsilon_{n+1}$. So (b) $\Rightarrow\left(c^{\prime}\right)$.

Now assume that (b) holds but (c) does not. Let $\left\{\delta_{n} \mid n=1,2, \ldots\right\}$ be a 
strictly decreasing sequence of real numbers such that $\delta_{1}<\epsilon$ and $\lim _{n \rightarrow \infty} \delta_{n}$ $>0$. We shall obtain a contradiction by constructing a sequence $\left\{\mu_{n} \mid n=1,2, \ldots\right\}$ in $K$ and a sequence of open subsets $\left\{U_{n} \mid n=1,2, \ldots\right\}$ such that

(4) $\mu_{n}\left(U_{n}\right)>\delta_{n-1}$, where $\delta_{0}=-1$,

(5) for $n \neq m, \bar{U}_{n} \cap \bar{U}_{m}=\varnothing$,

(6) if $Q$ is a compact subset of $E \sim \bigcup_{k=1}^{n} \bar{U}_{k}$, then there is a $\mu \in K$ such that $\mu\left(\left(E \sim \bigcup_{k=1}^{n} \bar{U}_{k}\right) \sim Q\right)>\delta_{n}$.

Let $U_{1}=\varnothing$, and let $\mu_{1}$ be any member of $K$. Suppose $\mu_{1}, \ldots, \mu_{n}$ and $U_{1}, \ldots, U_{n}$ have been chosen in accordance with (4), (5), and (6). The empty set is a compact subset of $E \sim \bigcup_{k=1}^{n} \bar{U}_{k}$, and so there is a $\mu_{n+1} \in K$ such that $\mu_{n+1}\left(E \sim \bigcup_{k=1}^{n} \bar{U}_{k}\right)>\delta_{n}$. Let $F$ be a compact subset of $E \sim$ $\bigcup_{k=1}^{n} \bar{U}_{k}$ such that $\mu_{n+1}(F)>\delta_{n}$. By $\left(c^{\prime}\right)$ there is an open set $V \supset F$ such that $\mu(V \sim F)<\delta_{n}-\delta_{n+1}$ for all $\mu \in K$. Let $U_{n+1}$ be an open set such that $F \subset U_{n+1} \subset \bar{U}_{n+1} \subset V \sim \bigcup_{k=1}^{n} \bar{U}_{k}$. Then $\mu_{n+1}\left(U_{n+1}\right) \geq \mu_{n+1}(F)>\delta_{n}$, and so conditions (4) and (5) are satisfied. Let $Q$ be a compact subset of $E \sim$ $\bigcup_{k=1}^{n+1} \bar{U}_{k} \cdot Q \cup F$ is a compact subset of $E \sim \bigcup_{k=1}^{n} \bar{U}_{k}$, and so there is a $\mu \in K$ such that $\mu\left(\left(E \sim \bigcup_{k=1}^{n} \bar{U}_{k}\right) \sim(Q \cup F)\right)>\delta_{n}$. Since

$$
\left(E \sim \bigcup_{k=1}^{n} \bar{U}_{k}\right) \sim(Q \cup F) \subset\left(\left(E \sim \bigcup_{k=1}^{n+1} \bar{U}_{k}\right) \sim Q\right) \cup\left(\left(E \cap \bar{U}_{n+1}\right) \sim F\right),
$$

we have $\delta_{n}<\mu\left(\left(E \sim \bigcup_{k=1}^{n+1} \bar{U}_{k}\right) \sim Q\right)+\delta_{n}-\delta_{n+1}$, and so $\mu\left(\left(E \sim \bigcup_{k=1}^{n+1} \bar{U}_{k}\right) \sim Q\right)$ $>\delta_{n+1}$. The sequences thus constructed constitute a contradiction.

(c) $\Rightarrow$ (d): Let $\epsilon>0$, and assume $\left\{E_{n} \mid n=1,2, \ldots\right\}$ is a disjoint sequence of Borel sets. Let $H_{n}=\bigcup_{k=n}^{\infty} E_{k}$, and let $F_{n}$ be a compact subset of $H_{n}$ such that $\mu\left(H_{n} \sim F_{n}\right)<2^{-n} \epsilon$ for all $\mu \in K$. Since $\bigcap_{n=1}^{\infty} F_{n}=\varnothing$, there is an integer $N$ such that $\bigcap_{n=1}^{N} F_{n}=\varnothing$. Now $H_{N}=H_{N} \sim \bigcap_{n=1}^{N} F_{n} \subset$ $\bigcup_{n=1}^{N}\left(H_{n} \sim F_{n}\right)$, and so for $\mu \epsilon K, \mu\left(H_{N}\right) \leq \Sigma_{n=1}^{N} 2^{-n} \epsilon<\epsilon$. So $\lim _{n \rightarrow \infty} \mu\left(E_{n}\right)$ $=0$ uniformly for $\mu \in K$.

(d) $\Rightarrow$ (a): We first construct a $\lambda \in M(X)^{+}$such that every member of $K$ is $\lambda$-continuous and then show that $K$ is necessarily uniformly $\lambda$-continuous. If $\lambda, \mu \in M(X)^{+}$, let $\mu=\mu_{\lambda}+\mu_{\lambda}^{\prime}$ be the Lebesgue decomposition of $\mu$ with respect to $\lambda$, where $\mu_{\lambda}$ is $\lambda$-continuous and $\mu_{\lambda}^{\prime}$ is $\lambda$-singular. We first show that, given $\epsilon>0$, there is a $\nu \in M(X)^{+}$such that $\mu_{\nu}^{\prime}(X)<\epsilon$ for all $\mu \epsilon K$. Suppose there is an $\epsilon>0$ for which there is no such $\nu$. Let $\epsilon_{1}=0$ and $\epsilon_{n}=$ $\epsilon$ if $n \geq 2$. We now construct a sequence $\left\{\nu_{n} \mid n=1,2, \ldots\right\}$ in $K$ such that

(7) $\nu_{n}(X) \geq \epsilon_{n}$,

(8) if $n \neq m, \nu_{n}$ and $\nu_{m}$ are mutually singular. Let $\nu_{1}=0$, and suppose $\nu_{1}, \ldots, \nu_{n}$ have been chosen to satisfy (7) and (8). Let $\nu=\sum_{k=1}^{n} \nu_{k}$. By 
as sumption, there is a $\mu \in K$ such that $\mu_{\nu}^{\prime}(X) \geq 6$. Let $\nu_{n+1}=\mu_{\nu}^{\prime}$. Since $0 \leq \nu_{n+1} \leq \mu \in K, \nu_{n+1} \in K$, and the sequence $\left\{\nu_{n} \mid n=1,2, \cdots\right\}$ satisfies (7) and (8). If $n \neq m$, let $E_{n, m}$ be a Borel set such that $\nu_{n}\left(E_{n, m}\right)=\nu_{n}(X)$ and $\nu_{m}\left(E_{n, m}\right)=0$. Let $H_{n}=\bigcap_{m=1 ; m \neq n}^{\infty} E_{n, m}$, and let $G_{n}=H_{n} \sim$ $\left(\bigcup_{m=1 ; m \neq n}^{\infty} H_{m}\right)$. Now $\nu_{n}\left(H_{n}\right)=\nu_{n}(X)$, and $\nu_{n}\left(H_{m}\right)=0$ if $m \neq n$. So $\nu_{n}\left(G_{n}\right)$ $=\nu_{n}(X) \geq \epsilon_{n}$ for all $n$. Since $\left\{G_{n} \mid n=1,2, \ldots\right\}$ is disjoint, we have a contradiction. We can therefore find, for each integer $n$, a $\lambda_{n} \in K$ such that $\mu \epsilon$ $K \Rightarrow \mu_{\lambda_{n}}^{\prime}(X)<2^{-n}$. Let $\lambda=\sum_{n=1}^{\infty} 2^{-n} \lambda_{n}$.

Now let $\lambda$ be any member of $M(X)^{+}$such that every member of $K$ is $\lambda$-continuous. Suppose $K$ is not uniformly $\lambda$-continuous. There is an $\epsilon>0$ such that, for any integer $n$, there is a Borel set $E_{n}$ such that $\lambda\left(E_{n}\right)<2^{-n}$, and a $\mu_{n} \in K$ such that $\mu_{n}\left(E_{n}\right) \geq \epsilon$. Let $H_{n}=\bigcup_{k=n}^{\infty} E_{k}$. Now $\lambda\left(H_{n}\right)<2^{-n+1}$ for all $n$, and so $\lim _{n \rightarrow \infty} \mu_{k}\left(H_{n}\right)=0$ for all integers $k$. An easy inductive construction enables us to find a strictly increasing sequence $\left\{n_{k} \mid k=1,2, \ldots\right\}$ of integers such that for each $k$, we have $\mu_{n_{k}}\left(E_{n_{k}} \sim H_{n_{k+1}}\right) \geq \epsilon / 2$. Let $F_{k}=E_{n_{k}} \sim H_{n_{k+1}}$. Then $\left\{F_{k} \mid k=1,2, \ldots\right\}$ is a disjoint sequence of Borel sets, and $\mu_{n_{k}}\left(F_{k}\right) \geq \epsilon / 2$, a contradiction which completes the proof. Q.E.D.

It should be noted that the only place in which countable additivity is used is in the proof of $(d) \Longrightarrow(a)$, and this can be avoided. This seems to suggest that it might be possible to produce a more general result than Grothendieck's, which deals only with bounded and additive set functions. Additionally, the properties of the measures and sets used in the proof do not tend to be topological (such as the use of the finite intersection property in $(c) \Rightarrow(d)$ ), but more along the lines of "a finite union of compact sets is a compact set". This might indicate that, if $X$ is a set and $\Sigma$ a $\sigma$-ring of subsets, it might be possible to define regularity of set functions with relation to a given subclass of sets in $\Sigma$ in such a way as to produce a more general characterization of conditional weak compactness in $c a(X, \Sigma)$ or $b a(X, \Sigma)$ along the lines of the Theorem.

The author would like to thank the referee, whose suggestions and modifications added considerably to the final version of this paper.

\section{REFERENCE}

1. A. Grothendieck, Sur les applications linéares faiblement compactes d'espaces du type $C(K)$, Canad. J. Math. 5 (1953), 129-173. MR 15, 438.

DEPARTMENT OF MATHEMATICS, UNIVERSITY OF CALIFORNIA, LOS ANGELES, CALIFORNIA 90024

Current address: Department of Mathematics, California State University, Long Beach, California 90840 